\title{
A Field Study of Relatively Long Chopsticks Reduce Food Consumption
}

\author{
Hung-Ming Lin ${ }^{1}$,Hui-Hsi Hung ${ }^{2 *}$ \\ ${ }^{I}$ Department of Business Administration \& Institute of Management, Minghsin University of Science and \\ Technology, Hsinchu, Taiwan \\ ${ }^{2}$ School of Journalism and Communication, Minjiang University, Fujian, China \\ *Corresponding Author.
}

\begin{abstract}
In this study, we examined the influence of chopstick length on diners' food consumption in a Chinese box restaurant. Diners were randomly offered chopstick of two lengths $(23$ and $19 \mathrm{~cm})$, and after they finished eating, their lunch boxes were weighed to measure the actual quantity of food consumed in a real dining environment. The results indicated that diners who used the long chopsticks consumed a lower quantity of food and had fewer mouthfuls than did those who used the short chopsticks, suggesting that long chopsticks can help control the quantityof food consumed without affecting diners' satisfaction with the dining experience. Our findings and those of previous research prove that bite size is the main factor mediating the relationship between a utensil's physical characteristics and people's food consumption.
\end{abstract}

Keywords: chopstick length, food consumption, utensils

\section{Introduction}

Maintaining fitness and a healthy diet has become a growing concern because numerous nutrition experts have suggested that people maintain an appropriate level of daily caloric intake. However, most people lose track of the quantity of food they consume because of distractions engendered by digital devices such as smartphones, computers, and TVs [1,2]; and several environmental factors such as lighting, sound, and music [3,4] as well as tableware and utensil sizes [4-6]. In particular, tableware and utensils play a major role in food consumption. To clearly understand the relationship between dining utensils and food intake, one line of research has focused on the effect of tableware size on the quantity of food consumed[4-6,7].

Most studies have demonstrated that people who use larger utensils consume more food than do those who use smaller utensils[5,7]. For example,Wansink [7] revealed that people who used larger spoons consumed more food than did those who used smaller spoons. By contrast, Mishra et al. [5] reported that the use of a smaller fork resulted in a larger quantity of food consumed than did the use of a larger fork because people were willing to invest the effort to satiate their hunger. Different utensils appear to have different size effects on food consumption. Therefore, a clear common model must be developed to provide an improved overall understanding of how utensil size can affect the quantity of food consumed.

Chopsticks are among the major dining tools in Asia; nevertheless, research on utensil size seems to have paid little attention to the effects of chopstick dimensions on food consumption. Studies on chopstick size and shape have tended to focus on serving performance and subjective evaluations in order to provide recommendations for better use and provide guidance for mass production [8-10]. For example, Lin et al. [4] investigated the effect of chopstick length on food intake. They observed that eating duration played a crucial role in the quantity of food consumed. Compared with short chopsticks, long chopsticks reduced eating speed, which led to fewer mouthfuls; therefore, diners using long chopsticks consumed a smaller quantity of food overall. However, their study was conducted in a laboratory, and whether the observed effect would occur in a field study context requires further investigation. Accordingly, the purpose of the present study was toprovide data on the effect of chopstick length on actual food consumption during dining.

ISSN: 0010-8189

(C) CONVERTER 2021

www.converter-magazine.info 
To observe situational factors influencing diners' eating behavior that cannot be observed in a laboratory, we chose a Chinese box restaurant for data collection. When visiting a restaurant, customers pay money, time, and effort to effectively satiate their hunger. A meal in a restaurant entails a continuous consumption behavior rather than a single consumption behavior; accordingly, the restaurant setting could enable us to examine the influence of chopstick length on overall consumption. In addition, a certain number of people in Asia eat out, and restaurants are the most common settings for their meals.

This research was a field study usinganalysis of covariance, along with an In-Depth Interviews. The findings revealed that diners who used the longer chopsticks consumed a lower quantity of food and had fewer mouthfuls than did those who used the shorter chopsticks. The results indicated that people who want to lose weight can use long chopsticks for everyday meals to easily reduce their food intake. Moreover, restaurants may provide relatively long chopsticks to not only increase customers' satisfaction with the same quantity of food but also enhance customers' enjoyment of the meal.

\section{Field Study}

We conducted our field study at a popular Chinese box restaurant located in northern Taiwan near a university campus and having a history of more than 15 years. The restaurant has approximately 25 tables and serves both lunch and dinner meals. We obtained the restaurant owner's permission to conduct this study and observed customers' behavior in the restaurant. This restaurant offers a typical menu, and the main meal box items are fried chicken, fried fish, grilled pork, and grilled beef meals. Each meal box contains one main dish and three side dishes. Consumers can have meals in the restaurant or purchase food to take away.

Diners were provided with chopsticks of two lengths $(23$ and $19 \mathrm{~cm}$ ), and both chopstick types were made of stainless steel and had grooved tips for improved grip. Both chopstick types had the same thickness to ensure that they could grip the same volume of food during use. Chopsticks made of stainless steel are the most common in Taiwan. These two chopstick lengths could provide approximately optimal serving performance for food consumption $[8,10]$. The quantity of food consumed at different meal times may differ because of diners' relaxation or other factors. Accordingly, to minimize variations in the quantity of food consumed, we observed diners during only lunchtime over a period of 8 days. One of the coauthors of the study and a research assistant served as waitstaff in the restaurant. To minimize variations in meal weight, we selected the grilled pork meal - the most popular meal in the restaurant - as our target. For each meal, the two chopstick types were randomly offered to diners who ordered the grilled pork meal. Full meal boxes were weighed before being served to diners, and the average meal box weight was $500 \mathrm{~g}$. Each of the diners took the meal box ordered and a pair of chopsticks and then sat at a table to eat their meal. After each diner finished their meal and left the restaurant, the research assistant took the meal box left at the table and weighed it, recording the observed weight. The date and diner's gender were also noted.

\section{Results}

We observed 75 diners (35 used long chopsticks and40 used short chopsticks) and applied analysis of covariance (ANCOVA) to examine the influence of chopstick length on the quantity of food consumed, which was determined by the weight of food left in the box (less food left in the box indicated more consumption); we controlled for gender (male vs. female) in this examination. The ANCOVA results revealed that the long chopsticks resulted in a lower quantity of food consumed (i.e., more food left in the box) compared with short chopsticks $\left(\mathrm{M}_{\text {long }}=404 \mathrm{~g}\right.$ vs. $\mathrm{M}_{\text {short }}=$ $433 \mathrm{~g} ; \mathrm{F}(1,73)=6.199, \mathrm{p}<.05)$.

\section{Findings from In-Depth Interviews}

When we served in the restaurant, we also observed the diners' behavior. The quantity of food consumed appeared to be associated with mouthfuls and eating duration. To understand diners' thoughts and behavior, we randomly interviewed 30 diners, of whom 15 used long chopsticks and 15 used short chopsticks.

ISSN: 0010-8189

(C) CONVERTER 2021

www.converter-magazine.info 
To comprehend diners' thoughts about chopstick use, we asked them the following question after they finished their meals:"Do you feel that you could finish the meal faster when you used the chopsticks?"Ten diners who used the long chopsticks responded as follows:"I feel that it took me quite a while to finish the meal." By contrast, 12 diners who used the short chopsticks stated the following: "I feel that I could finish the meal rather quickly." Furthermore, we asked them the following question:"Do you feel that you took adequate mouthfuls when eating the meal?"Eleven diners who used the long chopsticks responded as follows: "I feel that I didn't take many mouthfuls while eating the meal." By contrast, 10 diners who used the short chopsticks stated the following: "I feel that I took adequate mouthfuls while eating the meal." We also asked them the following: "How long do you feel it took you to finish the meal?" Eleven diners who used the long chopsticks responded as follows: "I spent about 20 minutes."Moreover, 12 diners who used the short chopsticks stated the following:"I spent about 15 minutes."

Responses to follow-up questions further supported that eating duration and mouthfuls were key attributes of food consumption. Therefore, the qualitative data confirmed that diners who used the long (short) chopsticks spent a longer (shorter) time on their meals and took fewer (more) mouthfuls, resulting in a smaller (larger) quantity of food consumed.

\section{Discussion}

We observed that in a restaurant setting, diners using long chopsticks $(23 \mathrm{~cm})$ consumed a lower quantity of food than did those using short chopsticks $(19 \mathrm{~cm})$, which is consistent with the outcome that long chopsticks can cause people to slow down and increase their eating duration[4].

Long chopsticks reduce the number of mouthfuls and eating speed of diners because of their relatively poor pinching performance[8], which can help reduce caloric intake and prevent obesity[11] as well as increase satiation[12].

Pieces of evidence obtained from the current and previous research reveals that bite size is the primary factor mediating the influence of utensil size on diners' food consumption [4,5,7]. Previous studies on different types of utensils such as forks, spoons, and chopsticks seem to have provided inconsistent results. Nevertheless, using bite size as the mediator of the aforementioned influence, we found that these results are, in fact, highly consistent.

A previous study on the effect of fork size on the quantity of food consumed revealed that diners consume a lower quantity of food when they used smaller forks than they did when they used larger forks under controlled laboratory conditions [5]; this finding is consistent with that of previous research on the effect of spoon size on the quantity of food consumed [7]. However, the reverse would be the case in a restaurant context because diners in such a setting invest effort and money for their meals; this indicates that a smaller fork would give them the perception that they are not making adequate progress in finishing their meals, and this thus motivates them to eat more. According to our results, relatively long chopsticks do not cause diners to perceive that they are not making adequate progress in finishing their meals; diners appear to perceive long chopsticks to be larger than short chopsticks.

We conclude that regardless of the physical characteristics of utensils, bite size still inherently has a positive relationship with the quantity of food consumed. However, the relationship can vary under actual restaurant conditions because of the effect of moderators such as hunger as well as other psychological and social factors[13].

Overconsumption leads to obesity and other health problems. Our results provide practical implications for addressing overconsumption; specifically, the results suggest that people use long chopsticks to control the quantity of food consumed. For example, people who want to lose weight can use long chopsticks for everyday meals to easily reduce their food intake. Additionally, restaurants could offer long chopsticks to not only enhance customers' satisfaction with the same quantity of food provided but also increase customers' enjoyment of the meal.

Utensils play a crucial role in people's everyday food intake, which has a considerable influence on people's health and well-being; therefore, further understanding of the relationship between utensils' physical characteristics and people's food consumption is essential to contribute information for improved utensil use and production. Future ISSN: 0010-8189 
research can expand on this topic by studying the influence of a utensil's characteristics such as its handle shape, diameter, and material (wooden, bamboo, plastic, and metal) on a diner's consumption. Cross-sectional studies on the interaction of physical characteristics of utensils and tableware such as bowls and plates in terms of food consumption are also worth considering.

\section{References}

[1] E. Blass, D. Anderson, H. Kirkorian, T. Pempek, I. Price, M. Koleini, "On the road to obesity: Television viewing increases intake of high-density foods,"Physiology \& Behavior, vol.88, pp. 597-604, 2006.

[2] N. Stroebele, J. M.D. Castro, "Listening to music while eating is related to increases in people's food intake and meal duration,"Appetite, vol. 47, pp. 285-289, 2006.

[3] B. Wansink, K.V. Ittersum, "Fast food restaurant lighting and music can reduce calorie intake and increase satisfaction," Psychological Reports, vol. 111, pp. 228-232,2012.

[4] H.M. Lin, C.H. Lin, H.H. Hung, "Influence of chopstick size on taste evaluations,"Psychological Reports, vol. 116, pp. 381-387, 2015.

[5] A. Mishra, H. Mishra, T. M. Masters, "The influence of bite size on quantity of food consumed: afield study," Journal of Consumer Research, vol. 38, pp. 791-795, 2012.

[6] K.V. Ittersum, B. Wansink, "Plate Size and Color Suggestibility: The delboeuf illusion's bias on serving and eating behavior," Journal of Consumer Research, vol. 39, pp. 215-228, 2012.

[7] B. Wansink, K.V. Ittersum, J. E. Painter, "Ice cream illusions," American Journal of Preventive Medicine, vol. 31, pp. 240-243, 2006.

[8] R. Chen, M. Wang, J.H. Chang, "A study of the pinch performance and subjective satisfaction with chopsticks of different lengths," MATEC Web of Conferences, vol. 119, pp. 01017,2017.

[9] C.P. Ho, S.P. Wu, "Mode of grasp, materials, and grooved chopstick tip on gripping performance and evaluation," Perceptual and Motor Skills, vol. 102, pp. 93-103,2006.

[10] S.H. Hsu, S. P. Wu, "An investigation for determining the optimum length of chopsticks," Applied Ergonomics, vol. 22, pp. 395-400,1991.

[11] M. Shah, J. Copeland, L. Dart, B. Adams-Huet, A. James, D. Rhea, "Slower eating speed lowers energy intake in normal-weight but not overweight/obese subjects,"Journal of the Academy of Nutrition and Dietetics, vol. 114, pp. 393-402, 2014.

[12] A.M. Andrade, G.W. Greene, K.J. Melanson, "Eating slowly led to decreases in energy intake within meals in healthy women," Journal of the American Dietetic Association, vol. 108, pp. 1186-1191, 2008.

[13] N. Garg, B. Wansink, J. J. Inman, "The influence of incidental affect on consumers' food intake," Journal of Marketing, vol. 71, pp. 194-206, 2007. 